Note de lecture

Maroussia Raveaud, De l'enfant au citoyen : la construction de la citoyenneté à l'école en France et en Angleterre

Presses Universitaires de France, 2006, 207 p.

Marilyn Osborn

\title{
OpenEdition
}

Édition électronique

URL : http://journals.openedition.org/ries/248

DOI : 10.4000/ries.248

ISSN : 2261-4265

Éditeur

Centre international d'études pédagogiques

Édition imprimée

Date de publication : 1 décembre 2006

Pagination : 15-16

ISSN : 1254-4590

Référence électronique

Marilyn Osborn, «Maroussia Raveaud, De l'enfant au citoyen : la construction de la citoyenneté à l'école en France et en Angleterre », Revue internationale d'éducation de Sèvres [En ligne], 43 | décembre 2006, mis en ligne le 20 février 2012, consulté le 22 septembre 2020. URL : http://journals.openedition.org/ries/ 248 ; DOI : https://doi.org/10.4000/ries.248

Ce document a été généré automatiquement le 22 septembre 2020.

(c) Tous droits réservés 


\title{
Note de lecture
}

\section{Maroussia Raveaud, De l'enfant au citoyen : la construction de la citoyenneté à l'école en France et en Angleterre}

Presses Universitaires de France, 2006, 207 p.

\author{
Marilyn Osborn
}

\section{RÉFÉRENCE}

Maroussia Raveaud, De l'enfant au citoyen : la construction de la citoyenneté à l'école en France et en Angleterre, Presses Universitaires de France, 2006, 207 p.

1 Le livre de Maroussia Raveaud, inhabituel et stimulant, examine de façon approfondie la relation entre l'expérience de la scolarité et la citoyenneté. Il porte sur ce que les Anglais appellent souvent le « curriculum caché » dans les écoles primaires anglaises et françaises. L'auteur s'intéresse à la façon dont l'école socialise les jeunes enfants et à l'ensemble de l'expérience scolaire de ces enfants, s'attachant non seulement au savoir scolaire proprement dit, mais aussi à des attentes plus larges en termes de comportement et de façons d'être, ainsi qu'aux manières dont les jeunes enfants s'impliquent dans la vie de la classe et aux relations avec les adultes au sein de l'école.

2 L'argument central est que les systèmes scolaires des deux pays sont conçus pour mener à bien deux missions parfaitement distinctes. Chacun recouvre des attentes différentes de ce que signifie appartenir à un groupe et à une classe, des droits et devoirs attachés à cette appartenance, et enfin de ce que signifie "être bon élève ". Ainsi, l'idée française et républicaine d'intégration nationale et d'égalité des chances s'oppose au modèle holistique et multi culturel britannique, qui met l'accent sur l'enseignement individualisé et le développement de l'enfant considéré dans sa totalité. 
Il s'ensuit que les établissements scolaires transmettent des messages différents aux enfants concernant leur propre personne, mais aussi concernant leur statut comme membres de l'école. Il agit là, selon certains, des pierres angulaires à partir desquelles un modèle de citoyenneté peut se construire chez les enfants.

C'est une étude comparative qui s'appuie sur une tradition sociologique, philosophique et historique, mais dont le cœur est une étude ethnographique de douze écoles primaires. Raveaud a observé des enfants âgés de quatre à sept ans dans les deux systèmes scolaires. Elle est parvenue à établir des normes comportementales, des modes de pensée, d'action et de ressenti qui appartiennent en propre à chaque système éducatif. Ces différences marquées entre les écoles primaires françaises et anglaises ont également été mises en évidence par le programme de recherche de Bristol que j'ai dirigé avec des collègues (Osborn et al., 2003, Broadfoot et al., 2000, Broadfoot et Osborn, 1993), qui a étudié des enfants âgés de neuf à onze ans scolarisés en primaire et de douze à quatorze ans scolarisés dans le secondaire, dans les deux systèmes. Cependant, l'étude de Maroussia Raveaud est unique à maints égards. Elle a étudié la socialisation des enfants lors des premières années à l'école, elle a été observatrice dans la plupart de ces écoles et possède une vision unique, à la fois comme partie prenante et élément extérieur aux deux systèmes. Elle a débuté sa propre scolarité en Irlande du Nord, avant d'être scolarisée en France à l'âge de neuf ans. Son étude, reflet d'une immersion complète dans les deux systèmes, permet une richesse inhabituelle, faite d'observations détaillées et de pénétration sociologique.

4 L'auteur présente des arguments pertinents quant à la capacité des systèmes scolaires à refléter, au travers du filtre de la différence marquée entre cultures pédagogiques, une vue particulière, non seulement de l'apprentissage proprement dit, mais aussi de ce que signifie être élève puis, plus tard, citoyen. Elle utilise quelques exemples fascinants tirés d'incidents détaillés observés dans la salle de classe pour montrer comment tout cela fonctionne en pratique. Ces incidents - qui explicitent le contrôle que les enseignants exercent sur les mouvements et le comportement des élèves, la façon dont on attribue des responsabilités aux enfants et dont les conflits sont gérés en classe constituent, selon elle, la première étape dans la genèse des conceptions très différentes des sphères publiques et privées dans les deux pays. Dans les écoles françaises, les enfants font l'expérience d'une dissociation entre leurs personnalités publique et privée. En conséquence, l'idéal français de la citoyenneté suppose une mise à distance par l'individu de sa communauté de naissance - communauté locale, classe sociale, appartenance religieuse, identité ethnique et sexuelle - de manière à accoucher socialement d'un être universel "libéré " des structures et des préjugés locaux, agissant pour le bien commun. Au contraire, selon l'idéal anglais multiculturel de la citoyenneté, les enfants sont encouragés à se percevoir comme des individus uniques et comme des "entités globales », non comme des esprits-réceptacles n'existant que pour être emplis de connaissances et de compétences. Tout est fait pour encourager l'enfant à apprendre à être " quelqu'un de bien », tout d'abord au sein de sa propre famille, puis dans le cadre de la classe et de l'école, comme membre d'une communauté locale élargie, enfin comme membre d'une nation et comme citoyen du monde.

5 Ces arguments fascinants ne manquent pas d'emporter l'adhésion. Afin de parvenir à cette vision riche et détaillée, l'auteur a sacrifié quelques-unes des réflexions qui auraient pu naître de la mention des récentes réformes et des changements menés dans les deux pays en matière de politique éducative. Elle n'accorde que peu d'attention à 
l'impact de la mercatisation et de la mondialisation sur l'éducation, particulièrement en Angleterre, mais aussi, dans une large mesure, en France. En se concentrant principalement sur les écoles des quartiers défavorisés, elle aurait pu passer à côté des différences plus subtiles qui émergent entre les différents groupes sociaux dans les deux pays. Toutefois, ces limitations sont largement compensées par les résultats remarquables de l'étude.

6 Avant toute chose, cette étude apporte une contribution unique à une tradition relativement nouvelle de recherche comparative, qui réussit à passer avec aisance du micro au macro, de l'observation finement ajustée de la vie d'une classe à l'analyse éclairante des conceptions nationales de l'apprentissage et de la citoyenneté. Elle réussit à mettre en lumière ce qu'Alexander (2000) a appelé «l'interaction complexe des politiques, des structures, des cultures, des valeurs et de la pédagogie ». En ce sens, elle mérite de rencontrer un très large public de part et d'autre de la Manche.

INDEX

Index géographique : Angleterre, France, Royaume-Uni

\section{AUTEURS}

\section{MARILYN OSBORN}

Professeur, Graduate School Education, University of Bristol, Angleterre 